\section{Zwei nicht alltägliche (?) Kasuistiken einer Asthmaerkrankung}

\section{T. Hausen}

Essen
Zusammenfassung: Vorgestellt werden zwei Asthma-Kasuistiken aus einer allgemeinärztlichen Praxis. Nach langjährigem Verlauf mit wechselnder Intensität des Asthmas treten bei Frau K. ständig Exazerbationen auf, die eine Therapie mit systemischen Kortikoiden erfordern. Als Ursache wird eine NNR-Insuffizienz aufgedeckt, vermutlich iatrogener Genese. Nach diversen Therapieversuchen stellt sich als optimale Minimaltherapie mit resultierender völliger Beschwerdefreiheit die Kombination aus Fluticason und $3 \mathrm{mg}$ Methylprednisolon heraus. Kurz- und Gruppenschulung sowie Beschwerdefreiheit sind nicht in der Lage, die Patientin dauerhaft compliant zu halten. Herr PI. leidet seit frühester Kindheit unter einem allergischen Asthma, das 1982 eskaliert. Die Unverträglichkeit (Heiserkeit) von Beclomethasondiproprionat (BDP) und Budesonid machen eine Polypragmasie nötig, die erst mit Verträglichkeit von Flunisolid (Fls) und damit erfolgter Stabilisierung reduziert werden kann. Unter zuletzt alleiniger Inhalation von Fls und Salbutamol besteht Beschwerdearmut. Nach Wechsel auf Fluticason kann Salbutamol bei weiterer Stabilisierung zur Bedarfsmedikation reduziert werden. Je nach Intensität der Allergenbelastung treten jetzt nur noch im Februar/März Beschwerden auf, die eine Aktualisierung der Therapie in Dosis und Zahl der Therapeutika erforderlich machen. Im restlichen Jahresverlauf ist eine Minimaltherapie mit 1 Hub Fluticason à $250 \mu \mathrm{g}$ ausreichend, anhaltende Beschwerdefreiheit zu garantieren. Beide Patienten haben trotz Beschwerdefreiheit unter Minimaltherapie im Vergleich zu früheren Zeiten ihre Behandlung eigenmächtig reduziert bzw. beendet und sind erst durch Abfall der Peak-Flow-Werte bzw. durch Beschwerdeentwicklung wieder zur Therapietreue bekehrt worden.

Two Out-of-the Ordinary (?) Case Reports on Asthmatic Disease: This is a report on two cases of asthmatic disease presented to a general practitioner. After a prolonged course of many years during which the intensity of asthma varied, Ms K. suffered from constantly recurring exacerbations that required treatment with systemic corticosteroids. The reason was found to be an adrenocortical insufficiency suspected to be of iatrogenic origin. After various treatment attempts an optimal minimal therapy was found resulting in complete freedom from complaints, namely, a combination of fluticasone and $3 \mathrm{mg}$ methylprednisolone. However, brief instruction and group training

Pneumologie 2000; 54: 116 - 122

(c) Georg Thieme Verlag Stuttgart · New York ISSN 0934-8387 as well as freedom from complaints remained unsuccessful in keeping the patient compliant. - Mr PI had been suffering from allergic asthma since early childhood which escalated in 1982. Beclomethasone diproprionate (BDP) and Budesonid were not tolerated (hoarseness), so that polypharmacy became necessary. This could only be reduced after finding out that Flunisolid (Fls) was tolerated, so that stabilisation was achieved. Complaints were greatly reduced with sole inhalation of Fls and salbutamol. After having changed over to fluticasone it became possible to reduce salbutamol as stabilisation progressed, so that salbutamol was used only if required. Depending on the intensity of allergen exposure, complaints now occur in February/ March only, requiring updating of the therapy in respect of dosage and number of drugs used. During the remaining part of the year a minimal therapy using one stroke of $250 \mu \mathrm{g}$ fluticasone was found sufficient to ensure lasting freedom from complaints. Both patients reduced or terminated the treatment of their own accord despite freedom from complaints under minimal therapy and were reconverted to therapy compliance only after the peak flow values had dropped or the complaints had returned.

Frau Chr. K., Jahrgang 1949, gebürtige Australierin, leidet unter einem Asthma. Die Beschwerden, vorwiegend Husten und nächtliche Luftbeschwerden haben nach der 2.Schwangerschaft 1978 mit Nasenproblemen begonnen. Im Rahmen der Diagnostik ist eine Allergie auf Frühblüher, Hausstaubmilben und Tierhaare festgestellt worden. Das Schlafzimmer ist daraufhin saniert worden.

Bei der Erstvorstellung 1994 bestand ihre Therapie aus der topischen Anwendung sowohl eines $\beta$-Agonisten als auch Glukokortikoid, einem Theophyllinpräparat, Acetylcystein und DNCG. Trotz regelmäßiger (?) Einnahme bzw. Inhalation in der bisher verordneten Dosis klagte Frau K. darüber, dass immer wieder in Abständen von wenigen Wochen starke Luftnotanfälle auftreten würden, die nur mit hohen Dosen von Kortison abgefangen werden konnten. Zusätzlich klagte sie über ständige Hustenattacken, vor allen Dingen in den frühen Morgenstunden. Diese Verschlechterungen würden immer einige Zeit bis zu mehreren Wochen nach Absetzen der Kortisontabletten auftreten. Typische Trigger konnten nicht eruiert werden.

Die Untersuchung des basalen Kortisolspiegels klärte die Neigung zur Destabilisierung. Der basale Kortisolspiegel lag bei $0,1 \mu \mathrm{g} / \mathrm{dl}$. Der ACTH-Test, die Kortisolausscheidung im Urin 
Tab. 1 Gemessene Hormonparameter zur Differenzialdiagnostik der NNR-Insuffizienz

\begin{tabular}{lll}
\hline & Messwert & Referenzbereich \\
\hline ACTH im Serum & & $10-50 \mathrm{pg} / \mathrm{ml}$ \\
Kortisol im Serum (nüchtern) & $<0,1 \mu \mathrm{g} / \mathrm{dl}$ & $3,1-22,4 \mu \mathrm{g} / \mathrm{dl}$ \\
Kortisol 60 Minuten nach ACTH & $<0,1 \mu \mathrm{g} / \mathrm{dl}$ & $28,5-213,7 \mu \mathrm{g} / \mathrm{dl}$ \\
Kortisol im 24-Std-Urin & $1,7 \mu \mathrm{g} / \mathrm{dl}$ & $>20,0 \mu \mathrm{g} / \mathrm{dl}$ \\
\hline
\end{tabular}

(Tab.1) sowie wiederholte Messungen des basalen Kortisolspiegels bestätigten die Nebennierenrindeninsuffizienz. Wiederholte Gaben von Kortisonpräparaten mit großer supprimierender Potenz auf die Hypothalamus-NNR-Achse müssen als wahrscheinliche Schädigung angenommen werden. Eine Befragung zu zurückliegenden Therapien mit Kortison konnte zur Ursachenklärung nicht beitragen.

Nach ausführlichen Einzelschulungen wurde die Therapie primär mit den bisherigen Therapeutika nach Dosiskorrektur beibehalten. Als Ziel wurde ein stabiles Krankheitsbild bei möglichst geringer Dauertherapie vereinbart. Nach und nach wurde die Therapie auf eine Basistherapie mit einem topischen $\beta$-Agonisten, einem topischen (mittels Dosieraerosol über Spacer) und systemischen Glukokortikoid und Theophyllin reduziert. Durch extrem langsame Reduktionsschritte der systemischen Kortisondosis sollte versucht werden, die Körpereigenproduktion vielleicht doch noch einmal anzuregen. Bei diesen Bemühungen war die Kortisonangst der Patientin ein nachhaltiges Problem. Verständlicherweise war sie bestrebt, das Kortison möglichst schnell wieder „loszuwerden“. Diese Angst wurde zusätzlich durch die Diagnose einer deutlichen Osteoporose in der Densitometrie geschürt. Wiederholt musste sie deshalb von der Notwendigkeit einer langsamen Reduktion der Kortisondosis bis zum Absetzen überzeugt werden.

Verschiedene Versuche mit unterschiedlicher Geschwindigkeit beim ausschleichenden Absetzen der systemischen Kortisontherapie waren leider zu Anfang erfolglos. Nach unterschiedlich langen Zeitintervallen, d.h. bis zu maximal 2-3 Wochen nach Absetzen der systemischen Glukokortikoidthe- rapie, kam es immer wieder zu Exazerbationen, die eine höhere Dosis systemischer Steroide erforderlich machten.

Nach Umstellung von Budesonid auf Fluticason (DA mit Spacer) stabilisierten sich die PEF-Werte zunehmend (Abb.1). Trotzdem kam es bei niedrigen Dosen des systemischen GKS immer wieder zu Exazerbationen. Selbst eine langsame Reduktion über mehr als drei Monate und bis zu $1 \mathrm{mg}$ Prednisolon führte erneut zur Verschlechterung des Befindens und Absinken der Peak-Flow-Werte. Eine nur leichte Dosisanhebung auf $4 \mathrm{mg}$ mit erneuter Reduktion konnte eine erneute Dekompensation nicht verhindern (Abb.1).

Die basalen Kortisolspiegel zeigten nach wie vor eine nahezu fehlende Eigenproduktion der Nebenniere und erhärteten die Diagnose einer irreversiblen NNR-Insuffizienz. Es galt jetzt, eine möglichst niedrige Dosis des systemischen GKS zu titrieren. Entsprechend den Empfehlungen in der Literatur konnte bei der iatrogenen NNR-Insuffizienz auf die Gabe eines Mineralokortikoids verzichtet werden [1]. Die Patientin wurde eingehend über die Problematik einer NNR-Insuffizienz einschließlich der Gefahren in Stresssituationen aufgeklärt und mit einem Kortisonausweis versorgt.

Es bedeutete eine erhebliche Aufklärungsarbeit, die Patientin davon zu überzeugen, dass sie mit ziemlicher Sicherheit lebenslang eine niedrige Dosis eines systemischen Glukokortikoids zu sich nehmen müsse, um ihr Asthma einigermaßen stabil zu halten und gleichzeitig den Bedarf des Körpers zu decken. Erst der zahlenmäßige Beleg, dass sie zu Zeiten eines instabilen Asthmas im Jahresverlauf eine wesentlich größere Menge Kortison zu sich genommen hatte, als jetzt zum Zeitpunkt eines beschwerdefreien Asthmaverlaufes war ein ausreichendes Argument.

Langsam aber sicher stellten sich zwischenzeitlich die Zeichen einer abnehmenden Aktivität des Asthmas ein (Abb. 1 u. 2), kenntlich an der zunehmenden Verbesserung der nächtlichen Hustenattacken, der Peak-Flow-Werte, den erreichbaren Dosen vor Exazerbation und den beschwerdefreien bzw. -armen Intervallen. Eine erfolgreiche und anhaltende Stabilisierung konnte dann endlich mit einer Minimaldosis von $3 \mathrm{mg}$ Methylprednisolon erreicht werden. $\mathrm{Zu}$ diesem Zeitpunkt benötigte die Patientin zur anhaltenden Stabilisie-

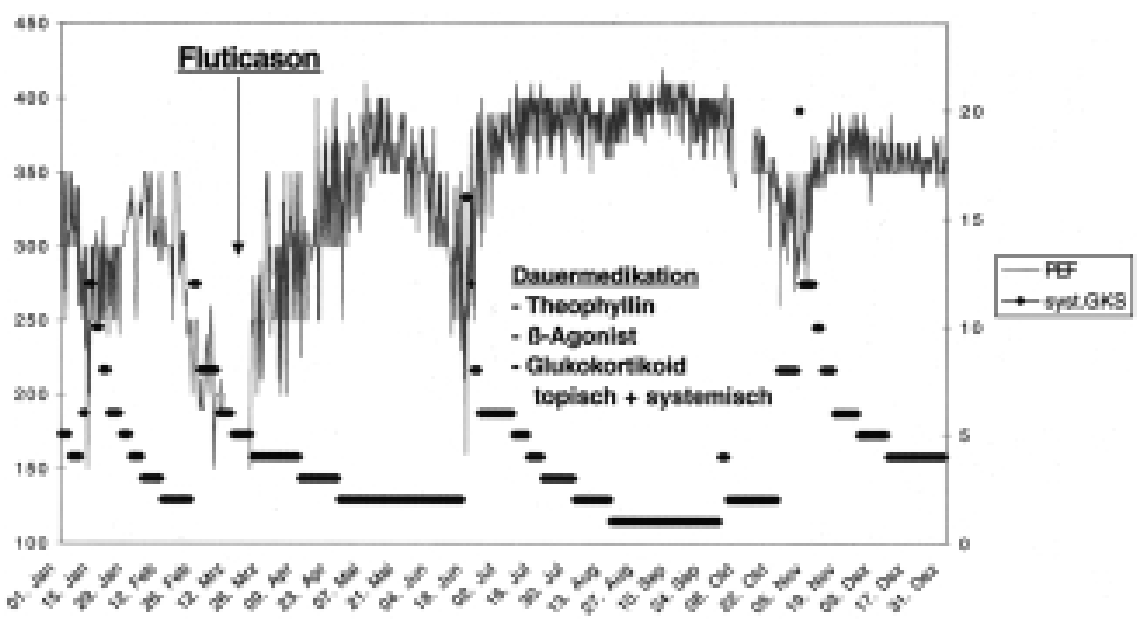

Abb. 1 Nach Umstellung des inhalativen Glukokortikoids auf Fluticason und langsamerer Reduktion der systemischen Kortisondosis stellt sich eine zunehmende Stabilisierung des Asthmas ein, kenntlich am Anstieg der Peak-Flow-Werte und Abnahme der Tagesschwankungen.

Skalierung links = Peak-Flow Werte in Liter, rechts Dosis $=$ Methylprednisolon in $\mathrm{mg}$. 


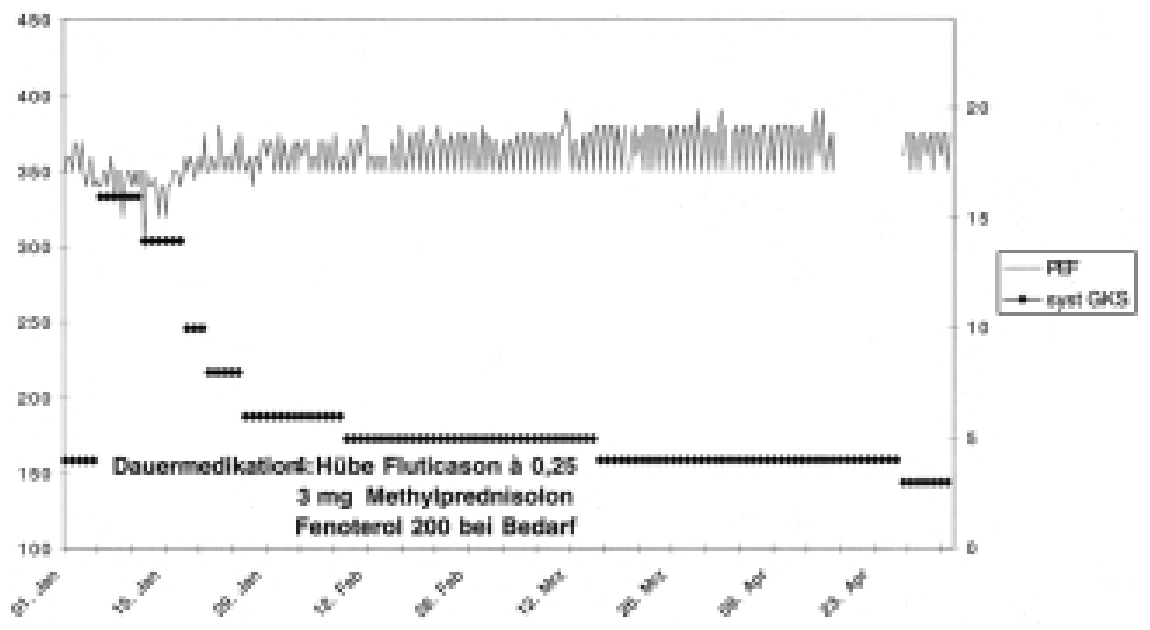

Abb. 2 Skalierung siehe Abb. 1. Die PeakFlow-Werte bleiben bei knapp 4 Hüben Fluticason à $0,25 \mathrm{mg}$, langsamer Reduktion der systemischen Dosis des GKS bis auf minimal $3 \mathrm{mg}$ Methylprednisolon und Fenoterol 200 bei Bedarf (im Verordnungszeitraum ca. 3 Hübe/Tag) anhaltend stabil.

rung neben dem systemischen GKS 4 Hübe Fluticason à $0,25 \mathrm{mg}$ und Fenoterol bei Bedarf. Im Verlaufe dieser Stabilisierung konnten sowohl die tägliche Inhalationsfrequenz reduziert als auch die Therapiekosten von anfänglich $18 \mathrm{DM}$ auf knapp 6 DM pro Tag gesenkt werden.

Im Verlaufe der Behandlung nahm die Patientin 1994 auch an einer Gruppenschulung teil. Bei weiteren Vorstellungen wurde die Problematik der Langzeittherapie mit Glukokortikoiden wegen der schlechten Erfahrungen wiederholt vorsorglich angesprochen, um die Patientin „bei der Stange“ zu halten. Trotz aller intensiven Beschäftigung mit der Patientin einschließlich Patientenschulung und Beschwerdefreiheit dank erfolgreicher Stabilisierung gestaltete sich die Führung der Patientin schwerer als erwartet. Immer wieder musste sie z.B. anlässlich von Rezeptnachforderungen auf vereinbarte Vorstellungen hingewiesen werden. Ende 1998 musste dann bei der Ermittlung des Jahres-Medikamentenverbrauches mit Schrecken festgestellt werden, dass die Patientin die Therapie im Verlaufe des Jahres eigenmächtig beendet hatte. Die letze Verordnung der Atemwegstherapeutika datierte vom Mai. Nur wenige Tage später, noch bevor Gelegenheit zur eigenen Aktivität bestanden hatte, stellte die Patientin sich vor und klagte erneut über nächtlichen Husten und leichte Luftnotanfälle. Sie gab jetzt auch mit Bedauern zu, in der anhaltenden Beschwerdelosigkeit geglaubt zu haben, dass sie die Therapie nicht mehr nötig gehabt hätte. Die Inhalation des Glukokortikoids hatte sie wegen anhaltender Beschwerdefreiheit eingestellt, das systemische Steroid in der zuletzt ermittelten Dosis von $3 \mathrm{mg}$ und die Therapeutika zur Behandlung der Osteoporose aber weiter eingenommen. Die Überprüfung der verordneten Dosen bestätigte diese Aussage.

Die Kontrollen belegten nach wie vor eine nahezu fehlende Eigenproduktion für Kortisol. Die Kontrolle der Densitrometrie ergab jetzt gegenüber dem Befund von 1997 auch eine deutliche Besserung der Osteoporose, nachdem die anfängliche Therapie mit Kalzium und Vitamin $\mathrm{D}_{3}$ um ein Biphosphonat erweitert worden war.

Herr L. Pl., Jahrgang 1940, leidet seit frühester Kindheit unter einer Rhinokonjunktivitis allergica und Asthma. Zum Zeitpunkt der Erstvorstellung 1983 hatte sich sein Asthma erst- malig dermaßen verschlechtert, dass er die Treppen zu seiner Wohnung kaum mehr steigen konnte. Sportliche Aktivitäten wie regelmäßiges Joggen und Radfahren, ja selbst Spaziergänge hatte er einstellen müssen.

Die Allergietestung (Ruhrlandklinik Essen) ergab im Prickund Intrakutantest stark positive Reaktionen auf Hausstaubmilbe, Hund, Katze, Pferd, Gräser, Bäume (Birke, Erle, Hasel, Eiche) und Roggenpollen, eine mittelstark positive Reaktion auf Alternaria und Schimmelpilzgruppe M11. Bei einem Gesamt-IgE von 374 U/ml war der RAST-Test auf Hausstaubmilbe fraglich positiv. Die Lungenfunktion zeigte eine leichte Erhöhung des Atemwiderstandes mit starkem Anstieg auf die Kontrolllösung, so dass eine bronchiale Provokation unterbleiben musste.

Die umgehende Sanierung der Umgebung, d.h. Abschaffung des Hundes innerhalb von 2 Tagen, Beseitigung aller Teppichböden und Ersetzen durch Keramikböden, Wechsel von Matratze und Bettwäsche waren nur von geringem Erfolg gekrönt. Auch eine dreijährige Hyposensibilisierung gegen Frühblüher erbrachte allenfalls eine vorübergehende Milderung der Beschwerden. Die intensive Reaktionsbereitschaft auf eine Allergenbelastung kam darin zum Ausdruck, dass die saisonalen Beschwerden bereits um die Vorweihnachtszeit begannen und bis Februar/März eskalierten.

Nur mit Hilfe aller (Salbutamol topisch und systemisch, DNCG, Theophyllin, Ketotifen, Antihistaminika und systemische Glukokotikoide; topische Applikation s. unten) der zu diesem Zeitpunkt vorhandenen Atemwegstherapeutika bei gleichzeitiger Applikation in verschiedenen Darreichungsformen und wechselnden Dosen waren die Atemwegsbeschwerden einigermaßen zu mildern. Dazu zählte auch ein kurzzeitiger aber erfolgloser Versuch mit parenteraler Gabe von Immunglobulinen.

Wiederholte Therapieversuche mit dem ersten Steroid zur topischen Anwendung (BDP) als Dosieraerosol unter Verwendung des spezifischen Spacers führten immer wieder zu massiver Heiserkeit bis hin $\mathrm{zu}$ völligem Stimmverlust und mussten beendet werden. Ein Therapieversuch mit Budesonid nach dessen Einführung unter Verwendung des dazugehöri- 
gen Spacers führte zu Besserung der Symptomatik, kenntlich auch am geringeren Verbrauch von Salbutamol und Sinken der Medikamentenkosten (Abb.6u.7), musste aber leider auch wegen zu starker Heiserkeit abgebrochen werden.

Bei dem für Nebenwirkungen offensichtlich empfänglichen Patienten traten regelmäßig die substanztypischen Nebenwirkungen auf. Bei dieser schon als Hilflosigkeit zu bezeichnenden Polypragmasie musste versucht werden, die aufgetretenen Nebenwirkungen mit anderen Medikamenten zu mildern, um die Therapie in dieser notwendigen Form beibehalten zu können. Zeitweilig musste der Patient unter diesen Bedingungen bis zu 9 verschiedene Therapeutika mehrfach täglich anwenden. Immer wieder mussten Therapieversuche wegen Auftreten von unerträglichen Nebenwirkungen abgebrochen werden; hierzu zählte auch ein Versuch mit einem lang wirksamen $\beta$-Agonisten (Serevent).

Erst mit Einführung des dritten inhalativen Steroids (Flunisolid über Spacer) trat eine Wende ein. Diese Substanz konnte toleriert werden, was erstmals die Möglichkeit zur vorsichtigen Reduktion der Therapie bot. Das systemische Glukokortikoid konnte ausschleichend abgesetzt werden. Theophyllin und die systemische Applikation des $\beta$-Agonisten konnten ebenfalls abgesetzt werden, bis für eine akzeptable Stabilisierung bei gleichzeitiger Beschwerdearmut nur noch eine regelmäßige Inhalation von Flunisolid und Salbutamol nötig war. Ausschließlich in den Frühjahrsmonaten musste die Therapie wegen der erhöhten Allergenbelastung mit zusätzlicher Rhinokonjunktivitis und der Verschlechterung des Asthmas vorübergehend in Dosis und Zahl der Substanzen gesteigert werden.

Der Wechsel von Flunisolid auf Fluticason (DA + Spacer) nach dessen Einführung führte zur weiteren Stabilisierung des Asthmas und Besserung der Obstruktion, was in den steigenden Peak-Flow-Werten zum Ausdruck kam. Erstmalig waren Beschwerden unter einer alleinigen Inhalation von Fluticason in jeweils aktualisierter Dosis und regelmäßiger Inhalation von Salbutamol nur noch in der Zeit von Februar/März zu verzeichnen.
Das mit dieser Verschlechterung verbundene Tal im PeakFlow-Protokoll konnte 1996 alleine durch Steigerung der Dosis des inhalativen Steroids von 4 auf 5 Hübe à $0,25 \mathrm{mg}$ abgeflacht werden (Abb. 3). Im weiteren Jahresverlauf stabilisierte sich das Asthma bei Peak-Flow-Werten um 550-570 l/ min, die Dosis konnte bis auf 2 Hübe Fluticason gesenkt werden. Im darauf folgenden Jahr 1997 blieben die Beschwerden unter der Therapie mit 4 Hüben Fluticason und 3-4 Hüben Salbutamol völlig aus. Auch dieser Patient glaubte bei anhaltender Beschwerdefreiheit, die Therapie nicht mehr nötig zu haben und unterbrach die regelmäßige Inhalation für wenige Wochen. Erneutes Auftreten von Beschwerden und gesunkene Peak-Flow-Werte waren Anstoß, die Therapie wieder aufzunehmen (Abb.4). Im weiteren Verlauf und anhaltender Stabilität war es trotzdem schwer, den Patienten zu einer ausschließlich bedarfsmäßigen Inhalation von Salbutamol zu überzeugen.

1998 kam es dann im Februar erstmalig wieder zu einer intensiven Beschwerdeentwicklung mit deutlichem PeakFlow-Abfall, die eine kurzzeitige Therapie mit einem systemischen Steroid erforderlich machte. Offensichtlich reichte im Vergleich zu 1997 die erneut angehobene Therapie auf $1 \mathrm{mg}$ Fluticason (= 4 Hübe à $0,25 \mathrm{mg}$ ) nicht aus, das Krankheitsbild stabil zu halten. Nach Beendigung der typischen Beschwerdezeit Februar/März konnte die Therapie langsam auf $1 \times 1$ Hub Fluticason à $0,25 \mathrm{mg}$ reduziert werden.

In der Absicht, 1999 einer erneuten Beschwerdeentwicklung durch rechtzeitige Therapiesteigerung vorzubeugen, wurde die Dosis von Fluticason Ende Dezember auf 2 und im Januar auf 4 Hübe pro Tag angehoben. Gleichzeitig erhielt der Patient zusätzlich täglich $10 \mathrm{mg}$ Montelukast, verbunden mit dem Wunsch, die Notwendigkeit einer systemischen Glukokortikoidtherapie verhindern zu können. Obwohl es auch jetzt zu einem kleinen Einschnitt in der Stabilität des Asthmas kam, verspürte der Patient jetzt keinerlei Symptome (Abb.5). Weder Salbutamol noch systemische GKS waren in dieser Saison erforderlich.

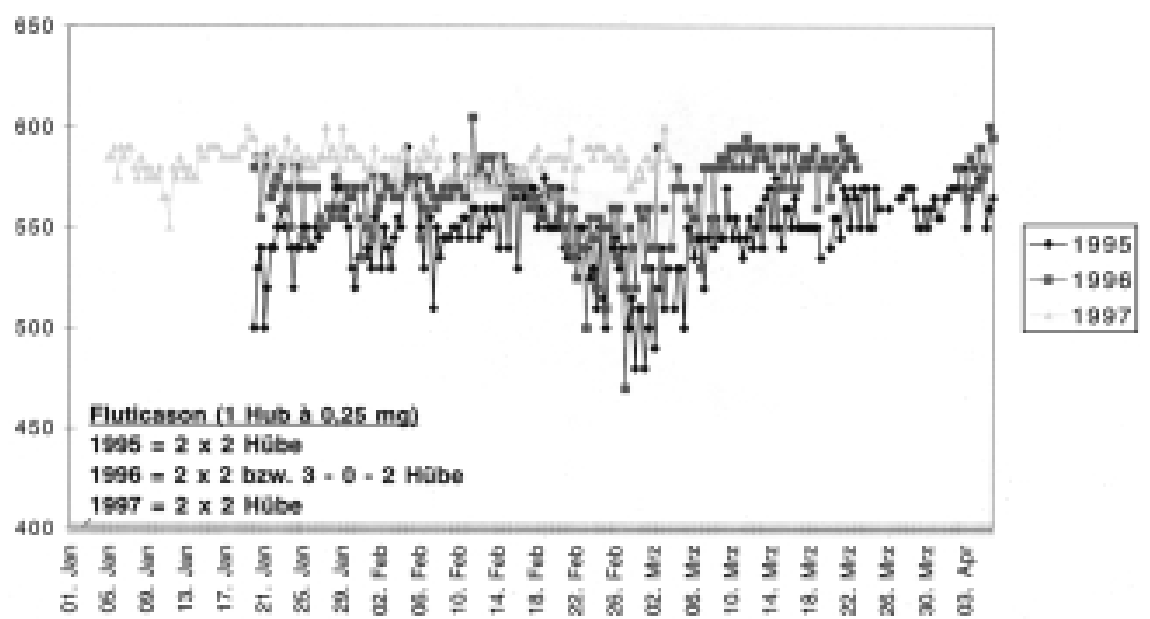

Abb. 3 Der Vergleich der Peak-Flow-Werte der Jahre 1995-1997 zeigt die zunehmende Stabilisierung des Asthmas, kenntlich am Anstieg der Peak-Flow-Werte bei gleichzeitiger Abnahme der Tagesschwankungen. Wahrscheinlich durch unterschiedliche Allergenbelastung kommt es auch trotz Therapieanhebung zu Peak-Flow-Abfällen zur typischen Jahreszeit. 


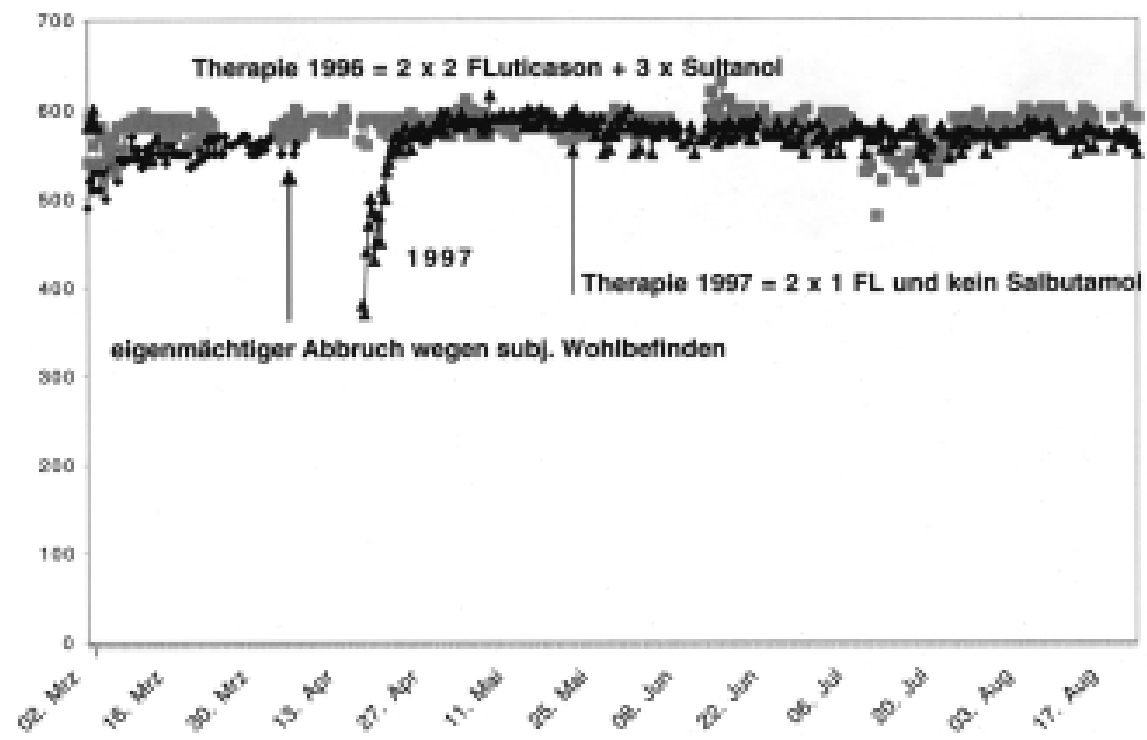

Abb. 4 Die anhaltende Stabilität des Asthmas verleitet auch diesen Patienten zur vorübergehenden Therapieunterbrechung. Beschwerdeentwicklung und Peak-Flow-Abfall veranlassen den Patienten, die Therapie ohne Rücksprache wieder aufzunehmen.

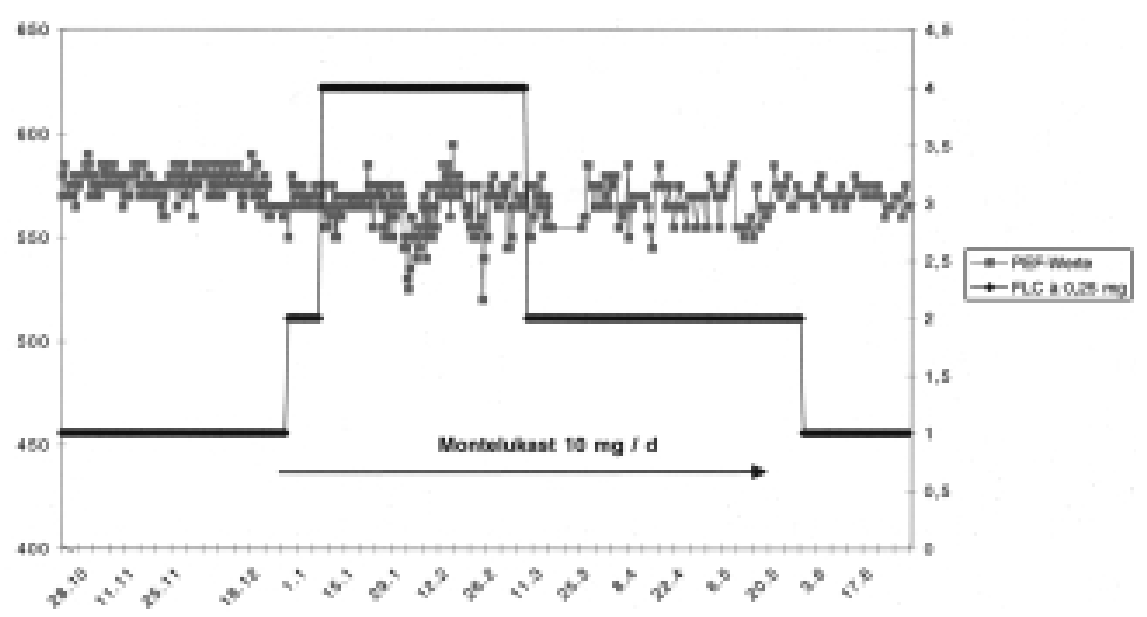

Abb. 5 Vorbeugende Dosissteigerung von Fluticason bei gleichzeitiger Applikation von Montelucast 1999. Bei nur leichtem PeakFlow-Abfall ohne subjektive Beschwerden wird Salbutamol oder Prednisolon in diesem Jahr nicht benötigt.

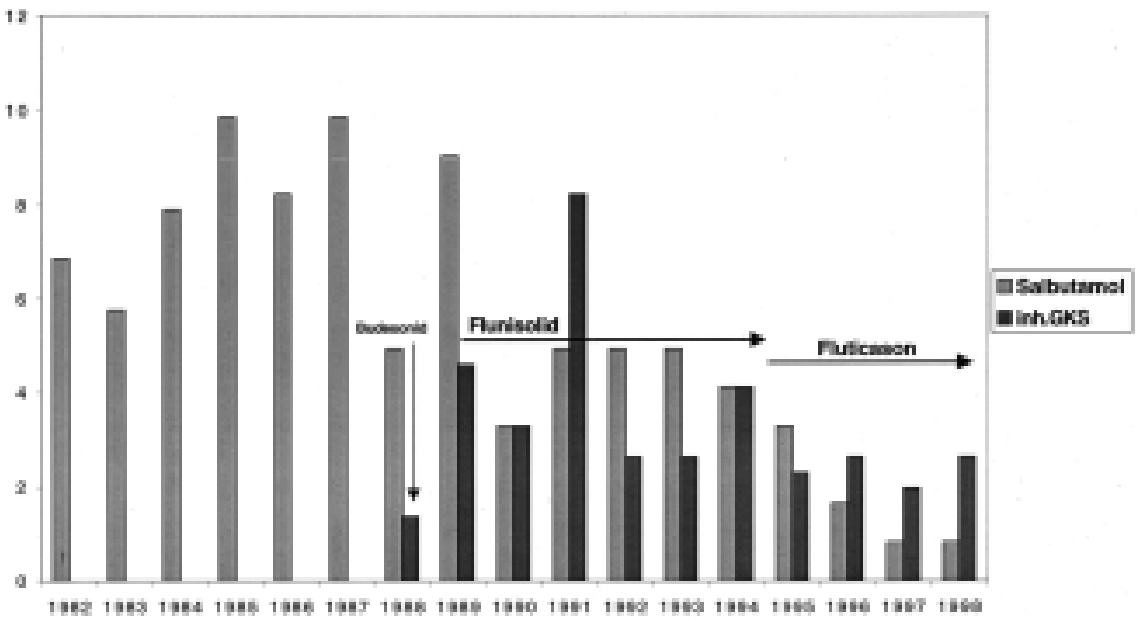

Abb. 6 Mit zunehmender Stabilisierung sank die Zahl der täglich notwendigen Inhalationen, berechnet am Verordnungsvolumen. 


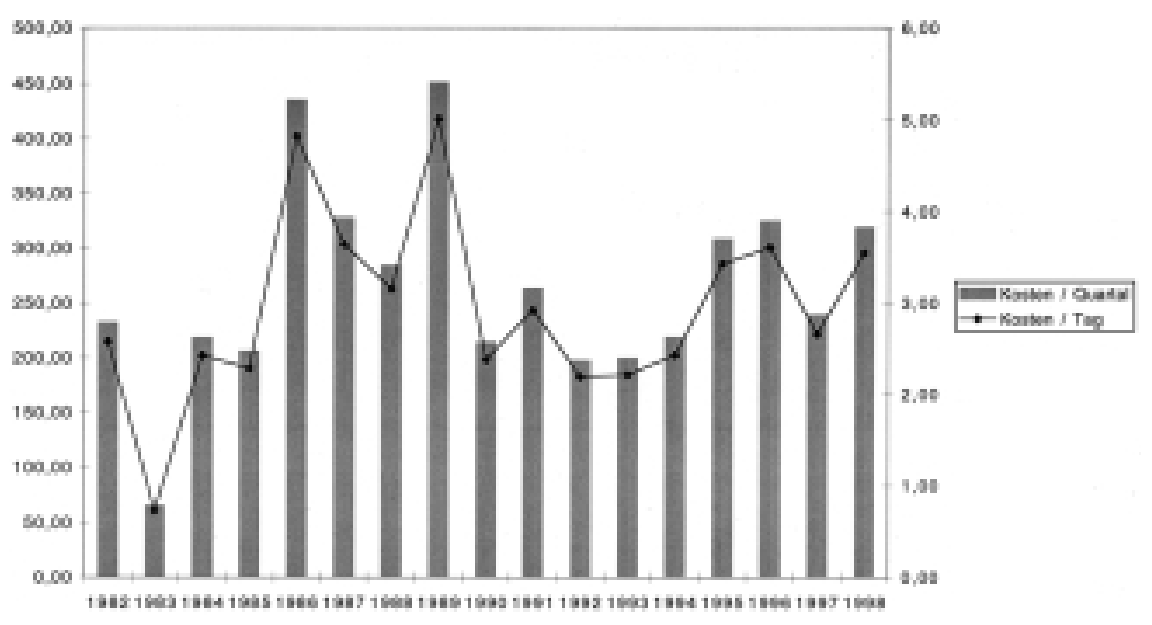

Abb. 7 Medikamentekosten.

Durch die zunehmende Optimierung der Therapie konnte die Belästigung durch die täglichen Anwendungen deutlich gesenkt werden. Die durchschnittliche Inhalationsfrequenz (Abb. 6), gemessen an den Medikamentenverordnungen, sank von maximal 14 auf 3,5 Hübe; es ist zu bedenken, dass Salbutamol kaum mehr nötig war, wegen des Verfallsdatums aber wiederholt verordnet werden musste und ebenfalls in diese Rechnung eingeflossen ist. Die tatsächlich aktuelle Inhalationsfrequenz liegt demnach noch niedriger. Die Medikamentenkosten sind in Abb. 7 wiedergegeben.

Der Patient hat Angebote zur Gruppenschulung stets abgelehnt, war aber durch die Kurzschulungen wiederholt in der Lage, eigenmächtig korrekt auf Veränderungen in seinem Krankheitsverlauf zu reagieren.

\section{Diskussion}

Die Erstdiagnose eines Asthmas erfordert die ganze Bandbreite der diagnostischen Möglichkeiten, um potenzielle Auslöser eines Asthmaanfalls zu ermitteln und diesen im weiteren Krankheitsverlauf möglichst effektiv vorbeugen zu können. Hier ist die Kompetenz des Pneumologen gefordert. Die anschließende Schulung hat eine Verhaltensänderung zum Ziel. Krankheitsakzeptanz, Therapiebereitschaft und Kortisonakzeptanz sind die Ziele einer Patientenschulung und Voraussetzung für eine erfolgreiche Langzeittherapie in der Praxis $[2,3]$.

Die Domäne des Hausarztes besteht darin, den Patienten während der Langzeittherapie zu führen, d.h. die optimale Basistherapie herauszufinden, regelmäßig zu aktualisieren und erfolgte Exazerbationen zu analysieren, um zukünftigen Exazerbationen vorzubeugen. Diese Aufgabe, verbunden mit der Notwendigkeit häufigerer Arzt-Patientenkontakte in kürzeren Abständen kann der Pneumologe, obwohl fachlich prädestiniert, aus vielen Gründen, oftmals alleine aus örtlichen Gegebenheiten nicht erfüllen. Kann der Hausarzt dieses Ziel allerdings alleine nicht erreichen, ist auch hier die partnerschaftliche Zusammenarbeit von Hausarzt und Pneumologe gefordert.
Die Kasuistik Pl.L. belegt eindrucksvoll die große therapeutische Potenz der topisch anwendbaren Glukokortikoide, gleichzeitig aber auch die Problematik, wenn auf deren Einsatz z.B. wegen Auftreten von Nebenwirkungen verzichtet werden muss. Das Auftreten von Heiserkeit sollte Anlass zu einem versuchsweisen Wechsel auf ein anderes topisches GKS sein, da diese Nebenwirkung nicht zwangsläufig bei allen Substanzen auftreten muss, bevor auf diese hochpotente Therapie verzichtet wird. Es lohnt auch immer, über eine Aktualisierung und damit Vereinfachung einer Therapie nachzudenken und in Zusammenarbeit mit dem Patienten zu versuchen. Unterschiede in der Wirkstärke der topischen GKS erlauben den Versuch mit einer höher potenten Substanz, um eine größere Stabilität zu erzielen. Die größere Stabilität erlaubt dann eventuell eine Reduktion der Dosis und/oder Anwendungsfrequenz und leistet einen wertvollen Beitrag zur Sicherung der Compliance.

Im geschilderten Fall hatte das wiederholte Auftreten von Heiserkeit bis zum Stimmverlust unter BDP und Budesonid trotz Inhalation über Spacer zur Folge, dass die Beschwerden des Patienten nur unter Einsatz von bis zu maximal 9 verschiedenen Therapeutika mit mehreren Anwendungen pro Tag einigermaßen erträglich gemacht werden konnten; dabei waren einige der Therapeutika erforderlich, um Nebenwirkungen zu mildern und den Einsatz der anderen Substanzen überhaupt erst zu ermöglichen. Erst mit Einführung des dritten GKS zur topischen Anwendung (Flunisolid) und Ausbleiben von Heiserkeit konnte eine ausreichende Stabilisierung erreicht werden, die das ausschleichende Absetzen aller anderen Substanzen bis auf Salbutamol und Flunisolid ermöglichte. Der versuchsweise Wechsel auf Fluticason, von dem eine noch größere Wirkpotenz in Studien nachgewiesen ist, ermöglichte eine weitere Therapiereduktion bis auf eine einmalige Tagesdosis bei gleichzeitig erhaltender Stabilität. Die regelmäßig bzw. bedarfsmäßig angewandte Inhalation von Salbutamol konnte bis auf wenige Ausnahmen unterbleiben. Gleichzeitig mit der weiteren Stabilisierung reduzierte sich die ehemals ganzjährige Symptomatik auf die Blütezeit der Hasel. Nur für diesen Zeitraum blieb ein Step-up mit Ausweitung der Therapie erforderlich. 
Das Streben nach anhaltender Stabilität und Einsparen von GKS, vor allen Dingen in der systemischen Applikationsform, lohnt immer den versuchsweisen Einsatz anderer Substanzen. Der frühzeitige Beginn einer begleitenden Therapie mit dem Leukotrienantagonisten (Montelukast) hielt die Peak-Flow Werte auf einem hohen Niveau und verhinderte das Entstehen von Beschwerden zur Zeit der Haselblüte. Die synergistische Wirkung erübrigte im Jahr 1999 eine systemische GKSTherapie.

Wider Erwarten aufgetretene Exazerbationen sollten einer intensiven Analyse unterzogen werden, um diesen in Zukunft durch geeignete Maßnahmen vorbeugen zu können. Das vorliegende Beispiel (Frau K.) weist auf die Notwendigkeit hin, in seltenen Fällen auch eine NNR-Insuffizienz und die damit verbundene „Schutzlosigkeit“ eines zu niedrigen Kortisolspiegels als Möglichkeit von Exazerbationen in Erwägung zu ziehen. An eine solche, seltene Möglichkeit muss auf jeden Fall gedacht werden, wenn bei sorgfältiger Anamnese kein Auslöser herauskristallisiert werden kann und gleichzeitig eine längere Asthmakarriere mit häufigen Exazerbationen und Notfallversorgungen und damit eher von unkontrolliertem Einsatz von systemischen GKS in höheren Dosen auszugehen ist.

Es ist bekannt, dass die Gefahr einer iatrogenen NNR-Insuffizienz bei Verwendung niedriger Dosen inhalativer GKS in der Langzeittherapie gering bzw. unwahrscheinlich ist; dies haben zahlreiche Untersuchungen bestätigen können. Bei Patienten unter einer regelmäßigen, systemischen GKS-Therapie wird eine NNR-Suppression im Vergleich zu alleiniger topischer Anwendung eher möglich bzw. erwartet [4,5]. Es existieren dagegen nur ganz spärliche Hinweise dafür, dass auch bei gelegentlichem Einsatz von systemischen GKS eine Schädigung der Hypothalamus-NNR-Achse zu befürchten ist [5]. Während wir bei wenigen und kurzen Phasen von selbst hohen Dosen systemischer GKS mit zusätzlich niedriger Suppressionspotenz von einer Erholung der NNR-Rinde ausgehen können [6], müssen wir bei wiederholten Therapiephasen auch eine Schädigung der Achse einkalkulieren [5] und wie im vorliegenden Fall als mögliche Ursache wiederholter Exazerbationen in Erwägung ziehen.

Zusätzlich weisen diese Kasuistiken darauf hin, dass die Applikationsformen offensichtlich nicht einfach gegeneinander austauschbar sind. Bei Frau K. hat die Fortsetzung der allerdings niedrig dosierten systemischen Anwendung nach Absetzen des topischen GKS und bei Herrn Pl. die alleinige topische Anwendung selbst nach Dosisanhebung nicht ausgereicht, eine Symptomentwicklung zu verhindern.

Es gibt immer wieder Patienten, bei denen wenige Einzelschulungen (Pl.L., [7,8]) ausreichen, um eine Stabilität des Krankheitsbildes bei gleichzeitiger Fähigkeit zum Selbstmanagement zu erzielen und bei denen sich die ausführliche und auch zeitaufwendige Gruppenschulung erübrigt. Eigene Untersuchungen haben allerdings auch belegen können, dass dieser Effekt bei vielen Patienten nur von relativ kurzer Dauer ist. Die Kombination aus Kurz- und Gruppenschulung einschließlich gezielter Nachschulungen hat sich als effektivste Möglichkeit erwiesen, eine länger dauernde Verhaltensänderung herbeizuführen $[2,3]$.
Die beiden vorgestellten Kasuistiken bestätigen die erwähnten Untersuchungsergebnisse, sind gleichzeitig aber auch Beleg dafür, dass eine intensive hausärztliche Betreuung einschließlich Kurz- und Gruppenschulungen keine Garantie für eine lang anhaltende gute Compliance sind. Trotz dieser Maßnahmen sowie besonders einer lang anhaltenden Beschwerdefreiheit bzw. -armut, die die zwei Patienten vorher nicht gekannt hatten, haben beide Patienten zumindest vorübergehend eigenmächtig ihre Therapie unterbrochen, bzw. reduziert. Herr P.L. unterbrach seine Therapie zur Zeit eines Auslandsurlaubs und wurde erst nach Rückkehr und erneutem Beginn von Peak-Flow-Messungen durch deren Abfall an die Notwendigkeit einer regelmäßigen Therapie erinnert. Frau K. brach die Inhalation des GKS ab, setzte jedoch die Therapie mit dem systemischen GKS fort. Erst das Auftreten von Beschwerden erinnerte sie an ihre Therapie und unterlassene Vorstellungen in der Praxis $[9,10,11]$.

\section{Literatur}

${ }^{1}$ Kaiser H, Kley HK. Cortisontherapie. Georg Thieme Verlag, 1997

${ }^{2}$ Hausen Th. Ambulante Kurzschulung im Vergleich zu Kombination aus Kurz- und Guppenschulung bei Patienten mit chronisch-obstruktiver Atemwegserkrankung. Präv-Rehab 1998; Jhg 10, Nr. 2: $83-88$

${ }^{3}$ Hausen Th. Patientenschulung - woran ist ein Langzeiteffekt abzulesen und wie lange hält dieser an? Pneumologie 1999; 53: $296-301$

${ }^{4}$ Krueger U, Spiecker H. Die Diagnostik der Nebenniereninsuffizienz bei steroidpflichtigem Asthma-bronchiale - Der CRH-Test im Vergleich zu Kortisol-Tagesprofil im Serum und Kortisol im 24-h-Urin. Pneumologie 1994; 48: 793 - 798

${ }^{5}$ Kos-Kudla B. Iatronic adrenal cortex failure in patients with steroid dependant asthma in relation to different methods of glucocorticoid treatment. Endocrine Regulations 32/2. 1998. $99-106$

${ }^{6}$ Zora JA, Zimmermann D, Carey TL, O'Conelly EJ, Yunginger JW. Hypothalamic-pituitary-adrenal axis suppression after shortterm, high-dose glucocorticoid therapy in children with asthma. $\mathrm{J}$ of Allergy and Clin. Immunology 1986; 77: 9 - 13

${ }^{7}$ Hausen Th. Langzeitbehandlung chronisch obstruktiver Atemwegserkrankungen im Spiegel der Kosten. MMW 1989; $131 \mathrm{Nr}$. 40: 721 - 722

${ }^{8}$ Hausen Th. Praktische Pneumologie (Pneumologische Kasuistiken unter besonderer Berücksichtigung von Lungenfunktion und Peak-Flow Messung). Kirchheim Verlag, 1996

${ }^{9}$ Hausen Th. Schulung von Atemwegskranken in der Praxis Evaluation und Information über Probleme bei der Evaluation. Präv-Rehab 1996; Jhg 8, Nr. 2: 47 - 52

${ }^{10}$ Karalus NC, Mahood CB, Dunn PJ, Speed JF. Adrenal function in acute severe asthma. New Zealand Medical J 1985; 98: 843 - 846

${ }^{11}$ Sakura N, Nishimura S, Kawahara Y, Yamaguchi S. Asthma as the first presenting symptom of complex glycerol kinase deficiency. Acta Paediatrica Scandinavica 1991; 80/6 - 7: 723 - 725

Dr. med. Thomas Hausen

Grafenstr. 52

45239 Essen 